Voix et Images

voixetimages

\title{
L’écrivain au théâtre
}

Lucie Robert

Volume 33, numéro 2 (98), hiver 2008

Michel Beaulieu

URI : https://id.erudit.org/iderudit/018282ar

DOI : https://doi.org/10.7202/018282ar

Aller au sommaire du numéro

\section{Éditeur(s)}

Université du Québec à Montréal

\section{ISSN}

0318-9201 (imprimé)

1705-933X (numérique)

Découvrir la revue

Citer ce compte rendu

Robert, L. (2008). Compte rendu de [L'écrivain au théâtre]. Voix et Images, 33(2), 156-164. https://doi.org/10.7202/018282ar d'utilisation que vous pouvez consulter en ligne.

https://apropos.erudit.org/fr/usagers/politique-dutilisation/ 


\author{
D R A M A T U R G I E \\ L'écrivain au théâtre \\ $+++$ \\ LUCIE ROBERT \\ Université du Québec à Montréal
}

Les pièces dont il est question plus loin marquent toutes bien nettement la présence de l'écrivain au théâtre. Il ne s'agit plus simplement de mettre en abyme une œuvre qu'un personnage lirait ou écrirait en scène ou de représenter un dialogue entre un auteur et un acteur (généralement en ce cas, plutôt une actrice), encore que ce modèle existe toujours et qu'il ait peut-être été comme un indice annonciateur. Il s'agit plutôt d'affirmer l'importance de l'écriture et la résurgence de l'existence textuelle (voire littéraire) du thêâtre. Plus nombreux qu'autrefois sont les auteurs dramatiques qui prennent la peine de publier leurs textes, souvent encouragés par les nouvelles maisons d'édition (dont l'existence même confirme ce fait), mais aussi plus nombreux sont les écrivains qui viennent ou qui reviennent au thêâtre. Si l'auteur ne fait toujours pas autorité sur scène (là n'est pas son lieu, qui appartient au metteur en scène), il s'impose de nouveau comme actant de première importance. Forcément, le retour de l'écrivain au théâtre (de l'écrivain tout court et non seulement celui que Michel Vaïs désignait sous le nom d' $\mathrm{d}^{\prime}$ écrivain scénique ${ }^{1}$ ») a pour conséquence un certain déplacement de l'écriture dramatique, non plus seulement centrée sur les besoins de la représentation (proposant un texte elliptique, écrit comme une partition et conçu pour laisser la plus grande place à l'acteur), mais également destinée à la lecture: «C'est important que le texte soit publié... et qu'on puisse voir clairement ta part de création ", dit Brigitte Haentjens à Louise Dupré, dans la conversation qui suit la publication de Tout comme elle ${ }^{2}$. C'est là quelque chose de neuf chez les metteurs en scène que cette humilité qui rend justice aux maîtres des mots.

Rien ne montre mieux l'importance qu'a récemment prise l'écriture dramatique que le simple fait que Robert Lepage ait entrepris de publier ses pièces. On aurait pu

$$
+++
$$

1 Michel Vaïs, L'écrivain scénique, Montréal, Presses de l'Université du Québec, 1978. 2 Louise Dupré, Tout comme elle, suivi d'une conversation avec Brigitte Haentjens, Montréal, Québec Amérique, coll. «Mains libres», 2006, p. 83. 
croire que la publication, il y a deux ans, de La trilogie des dragons ${ }^{3}$, avait été un accident de parcours; que Lepage avait publié ce texte, d'une part en réponse aux demandes insistantes des uns et des autres, d'autre part, parce qu'il en avait fini avec cette production et qu'elle avait atteint sa forme définitive. Toutefois, la parution récente de deux autres pièces, La face cachée de la lune ${ }^{4}$ et Le projet Andersen ${ }^{5}$, laisse supposer un virage chez l'acteur scénographe, qui se conçoit peut-être même désormais aussi comme un écrivain.

On sait, depuis le début de sa carrière, que la dramaturgie de Robert Lepage naît de la rencontre entre un thème et une scénographie, que le thème lui-même superpose toujours deux temps: un passé (un lieu, un personnage ou une œuvre source) et un présent (tout entier concentré dans un personnage de créateur en action), que le tout s'inscrit dans une démarche essentiellement autobiographique, et que celle-ci est à la fois fragmentaire (c'est-à-dire qu'elle ne propose aucune vision d'ensemble de la « vie») et menteuse (c'est-à-dire qu'elle entremêle les expériences vécues et la fiction). Quant à la scénographie, celle-ci a fait l'objet de tant de commentaires, qu'il n'est peutêtre pas nécessaire d'y revenir ici, sinon pour rappeler son caractère déterminant, car c'est de sa réalisation que dépend l'existence même de la pièce. Aussi, chacun des textes dramatiques que publie Lepage est-il accompagné d'une description précise du mécanisme scénique qui a présidé à la représentation. Dans La face cachée de la lune, ce mécanisme est un mur pivotant à trois faces et à basculement vertical, dont le mouvement entraîne à la fois un changement de temps et de lieu dans la fiction. Dessins, croquis et photographies rendent ces images facilement accessibles au lecteur qui peut ainsi reconstituer de façon imaginaire le caractère visuel du spectacle. Dans Le projet Andersen, il s'agit d'un écran tendu comme une conque sur lequel sont projetées des images en deux dimensions (formant une toile de fond) et devant lequel est installé un système de rails, sur lesquels se déplacent des objets (accessoires, meubles, marionnettes ou mannequins). Un DVD, qui déploie avec précision la nature même de ce mécanisme scénographique et qui, en outre, rappelle la genèse et la réception du spectacle, est inséré dans la couverture. Toutefois, l'image, aussi ingénieuse ou brillante qu'elle soit, ne fait pas l'écrivain, qui doit aussi avoir la générosité d'abandonner son texte à la méditation d'autrui. Bien qu'il soit parvenu avec le temps à abandonner ses textes à d'autres acteurs (Vinci fut joué par Marc Labrèche; La face cachée de la lune et Le projet Andersen ont été repris par Yves Jacques), Lepage n'a encore jamais laissé un autre metteur en scène en prendre possession. D'une certaine façon, l'abondance des images (fixes ou vivantes) qui accompagnent la publication des deux textes montre la même résistance: si elles permettent une meilleure connaissance du spectacle, tel qu'il a été représenté, elles pourraient aussi avoir pour effet de restreindre les possibles de la lecture. En ce sens, le DVD, construisant un objet visuel séparé de l'objet textuel, n'est pas une mauvaise idée.

$$
+++
$$

3 Voir ma chronique: "Voyager pour souffrir», Voix et Images, vol. XXXI, n 1, automne 2005, p. 166-173. 4 Robert Lepage, La face cachée de la lune, préface d'André Brassard, Québec, L'instant même, coll. «L'instant scène», 2007, 77 p. Photos. 5 Id., Le projet Andersen, préface de Lars Seeberg, Québec, L'instant même, coll. «L'instant scène», 2007, 92 p. Illustré. Avec un DVD. 
Car la lecture, moins distraite par l'image, entend mieux les mots. Dans La face cachée de la lune, créée au Théâtre du Trident le 29 février 2000 [sic], ces mots sont portés par le personnage de Philippe, qui doit soutenir une thèse où il démontre «que les programmes d'exploration spatiale du Xxe siècle ont été motivés non par la curiosité et la soif de savoir, mais bien par le narcissisme» (16). Ainsi, la compétition entre les Américains et les Russes, dans la conquête de l'espace, serait comparable à «celle de deux frères cherchant continuellement dans le regard de l'autre un miroir pour y contempler leurs propres blessures, ainsi que leur propre vanité» (15). C'est donc la relation entre deux frères, Philippe et André, l'un étant un homme d'affaires prospère, l'autre, un étudiant un peu paumé qui multiplie les échecs et les actes manqués, qui sert de trame à la fiction, mais c'est l'histoire considérée du point de vue de Philippe, encombré par ses souvenirs (il est tombé malade à la mort de son père et il conserve encore les vêtements de sa mère décédée), qui occupe le premier plan. Se déploie ainsi un personnage qui ne parvient pas à assumer le présent ni à construire l'avenir ( «tu fais pas de place pour que les choses t'arrivent, Philippe» [28]) et dont l'activité est tout entière tournée vers l'espace car, outre la thèse (qui sera refusée), Philippe prépare une vidéo destinée à d'éventuels visiteurs extraterrestres (qui sera primée par une émission de télévision). Découpée en scènes brèves, présentant toujours un seul personnage à la fois (écrite pour un acteur seul en scène), la pièce superpose ainsi la destinée individuelle et l'aventure cosmique dans une vaine tentative de «réconcilier l'infiniment banal, l'infiniment petit avec l'infiniment grand, l'infiniment essentiel» (47) de même qu'elle s'interroge sur la place de celui qui vient après, ici le frère cadet, là les Russes : "comment tu fais pour trouver la motivation de continuer à vivre quand t'as accompli de grandes choses mais que, bon, l'Histoire va se souvenir de toi comme d'un numéro deux, comme de quelqu'un qui a manqué sa chance?» (47)

Le projet Andersen est construit de la même manière: elle est toujours destinée à un acteur seul en scène, qui doit jouer plusieurs personnages, et elle est découpée en petites scènes qui se terminent toutes par un punch, une phrase incisive qui confirme l'enjeu de ce qui vient d'advenir et, surtout, qui soutient le rythme en marquant le tempo. Créée au Trident en février 2005 (une année non bissextile), la pièce a obtenu le Prix H. C. Andersen d'Odense, décerné «à une personne qui créerait une nouvelle œuvre inspirée par Andersen et ses contes » (8). Cette circonstance extratextuelle trouve un écho dans la fiction même, qui met en scène un jeune auteur québécois (par ailleurs albinos), qui doit adapter un conte d'Andersen, La dryade, pour l'Opéra Garnier de Paris. Lui font face (si l'on peut dire) un administrateur de l'Opéra Garnier et un jeune immigrant d'origine marocaine, qui travaille dans un peep-show où il surveille (et nettoie) les cabines. Ainsi, installé provisoirement à Paris, Frédéric découvre les diverses facettes de la ville : l'univers bourgeois de l'Opéra Garnier, où il travaille, l'univers sordide du peep-show, au-dessus duquel il habite, et la ville imaginaire du XIX siècle dont rêve le personnage d'Andersen. La rencontre entre ces trois univers n'est d'ailleurs pas sans provoquer quelques tensions : l'Opéra est perturbé par la grève des éboueurs et concierges, entraînée par l'arrestation d'un jeune arabe; les producteurs du spectacle (français, anglais et danois) auraient préféré un spectacle pour enfants, à partir d'un conte plus connu; 
les dealers sont à la recherche de Didier, dont Frédéric occupe l'appartement. La catastrophe est imminente et quand l'incendie se déclare, on sent bien que Frédéric en est arrivé à confondre sa vie et celle de l'écrivain danois, qui craignait le feu plus que tout au monde: «je me suis dit, ironiquement, que tout allait se terminer cruellement, comme dans un conte d'Andersen, où les êtres humains qui ont de trop grands désirs et de trop grandes ambitions sont toujours punis et où seuls les animaux sont heureux et ont beaucoup d'enfants" (92). Sans doute était-il grand temps que Lepage nous intéresse à autre chose qu'à ses images.

En réalité, l'univers imaginaire de Robert Lepage n'est pas si éloigné de celui des autres dramaturges, parmi lesquels des écrivains plus convaincus de leur place et de leur rôle, comme Wajdi Mouawad, dont la plus récente pièce, Assoiffés ${ }^{6}$ créée au Théâtre Lionel-Groulx de Sainte-Thérèse le 12 octobre 2006, dans une mise en scène de Benoît Vermeulen -, imbrique elle aussi plusieurs niveaux de fiction, dont elle brouille les frontières, et dont l'un est lui-même une histoire, écrite par l'un des personnages. Comme dans les autres pièces de Mouawad (Littoral, Incendies, Forêts), Assoiffés procède d'un événement singulier: on a découvert, au fond du SaintLaurent, deux corps «enlacés depuis si longtemps que leurs cadavres se sont fondus l'un dans l'autre» (13). La découverte a lieu en 2005, la mort remonterait à 1991, mais la pièce se déroule en 2006, quand l'anthropologue judiciaire chargé de l'enquête rend son rapport. L'un des cadavres est celui de Sylvain Murdoch dont la crise d'adolescence (en 1991) ouvre la pièce: «je ne me tairai pas, calvaire!» (9) L'autre est celui d'une inconnue. La cause du décès est vraisemblablement le suicide. Or, l'anthropologue judiciaire (Boon est son surnom) est également le frère de JeanRené qui était dans la même classe à l'école que Murdoch. Un jour, Boon, qui faisait les devoirs de son frère, apprend de Murdoch qu'il doit faire une enquête sur la beauté. Il écrit alors pour Jean-René une pièce de théâtre dont le personnage principal, nommé Norvège, est une fille qui refuse de sortir de sa chambre: «La sortie de Norvège allait être mon devoir sur la beauté.» (23) Mais Jean-René n'aime pas la pièce, dont il passe néanmoins pour avoir été l'auteur, mettant ainsi un terme à la carrière d'écrivain de son frère. Si l'on ignore ce que fut exactement la réaction de Murdoch, devant la représentation, l'on sait néanmoins qu'il s'est tu ce jour-là, au moment précis où le personnage de Norvège sortait de sa chambre. C'est elle d'ailleurs qui raconte être allée patiner avec lui jusqu'à ce que la glace se fende : «On s'est agrippés l'un à l'autre, à jamais le cœur de l'un dans le cœur de l'autre: personnage réel, personnage fictif.» (37) Murdoch est mort, bien sûr, mais Norvège est toujours là, car un personnage de fiction peut-il mourir, même s'il laisse un cadavre derrière lui ? Et que reste-t-il encore du personnage réel, quand l'auteur du personnage fictif

$$
++
$$

6 Wajdi Mouawad, avec la collaboration de Benoît Vermeulen, Assoiffés, Montréal/Arles, Leméac/Actes Sud, coll. «Papiers», 2007, 39 p. 
est aussi l'anthropologue chargé de l'enquête? Le vraisemblable lui-même se détraque, dans une construction qui frôle le baroque, mais peut-être aussi est-elle un peu convenue et pas entièrement dénuée d'effets de mode.

Larry Tremblay fait plus que frôler le baroque. Il s'y plonge avec volupté avec cette Histoire d'un cour ${ }^{7}$, dont il faut prendre le titre à la lettre. Dans une salle d'opération, où l'on s'apprête à le transplanter, le Cœur parle et proteste, affirmant qu'il ne lui reste que 4235 battements à offrir et que, par conséquent, sa vie sur terre est presque terminée. On peut croire que 4235 battements couvrent à peu près la durée d'une soirée au théâtre, de sorte que la fin est prévisible : le Cœur va s'arrêter. Entretemps tout de même, il aura conté sa vie, à commencer par sa naissance dans le corps d'Henri, sa première transplantation dans le corps d'Alejandro (qui le rejette) et une seconde transplantation, dans le corps d'un clown, nommé Léonard. Derrière lui s'agitent les personnages de son récit, parce que les choses ne sauraient être simples et rien n'arrive par hasard. Car Henri, né le jour même de l'assassinat de John Lennon, aurait voulu être chanteur comme lui. Il écrit d'ailleurs, mais il doit aussi s'occuper de sa fille Zoé, dont la mère l'a quitté quelque temps plus tôt. Il meurt juste au moment où une compagnie de disques accepte ses chansons. Zoé pourra tout de même poursuivre ses études en tirant profit des droits d'auteur. C'est ainsi qu'elle rencontre Alejandro, dont elle se refuse de tomber amoureuse, puisque ce serait pour elle comme un inceste. Elle apprendra trop tard que, en réalité, Henri n'était pas son père et que sa mère, Lucy, était tombée amoureuse de Léonard, un soir qu'il jouait Roméo et Juliette. C'était avant qu'il ne devienne clown à Las Vegas, où Zoé part le retrouver... La dramaturgie de Larry Tremblay hésite ainsi entre ce type de fantaisies - celle-ci une pièce pour acteurs et marionnettes, créée par le Théâtre Incliné au Périscope en mars 2006 - et les genres plus sérieux.

Mais peut-on vraiment parler de genre sérieux, à propos de La hache ${ }^{8}$, long monologue d'un professeur de littérature qui débarque chez un étudiant pour lui remettre la copie de son travail, mais créé à deux personnages (l'étudiant est néanmoins resté muet), au Théâtre de Quat'Sous, en avril 2006. Car le professeur compare son travail à la maladie de la vache folle. Nous sommes au milieu de la nuit et il pleut. Les premiers mots qu'il prononce sont pour le moins inquiétants: «Tu sais à quoi je pensais en venant ici? Je pensais aux vaches. On les assomme, on en fait des tas, on met le feu dedans. On appelle ça de la destruction. On détruit les vaches parce qu'on a peur qu'elles nous transmettent leur maladie. » (9) Encore plus inquiétantes sont les circonstances qu'il explique: «J'ai bu un bon coup avant de venir chez toi. J'ai mis le feu et je me suis enfui avec ma serviette de prof. Un homme seul avec un troupeau de vaches.» (29) Il a même croisé un ange dans un parc. De toute

$$
+++
$$

7 Larry Tremblay, L'histoire d'un cœur. Thêâtre, Carnières, Lansman éditeur, coll. «Nocturnes Thêâtre ", n 176, 2006, 46 p. 8 La hache est le premier des trois textes de Larry Tremblay qui composent Piercing, Paris, Gallimard, 2006, 158 p. 
évidence, le professeur dérape: son enseignement serait une maladie - il devrait donc être exterminé comme le sont les vaches folles - et il montre des instincts suicidaires. Pourtant, bien peu de temps s'est écoulé depuis son dernier cours et ne vient-il pas, à ce moment précis, remettre sa copie à l'étudiant? Le titre du monologue nous fournit un précieux indice sur la nature de cette copie et sur la conception de la littérature qui en découle. Toutefois, ce qui est particulier ici est bien la manière dont le professeur l'a reçue et les conséquences de ce qui paraît être une opération de séduction. Si l'étudiant le séduit, c'est qu'il possède ce que visiblement le professeur a perdu: le silence. "C'est ta façon à toi de parler: ton silence.» (55) Le silence serait ainsi l'absolu de la littérature plutôt que son degré zéro. Mais alors comment imaginer le monde? Quant à savoir lequel des deux personnages a séduit l'autre, la chose n'est guère possible, car nous n'avons ici qu'un seul énonciateur. C'est le professeur qui construit la figure de l'étudiant, qu'on n'entendra jamais. Il a fait sienne la position qu'il croit être celle de l'Autre: "Je suis une vache malade, contaminée, impure, dangereuse.» (63) Appel au suicide assisté pourrait-on dire. Le texte ne dit pas ce que fait l'étudiant, s'il va rester indifférent et appeler un hôpital ou s'il va faire ce qu'on lui demande.

Il est évidemment difficile de ne pas se souvenir de Jovette Marchessault, qui écrivait: «Ma mère était une vache. Avec moi ça fait deux ${ }^{9}$.» Toutefois, il faut aussi garder en mémoire que Marchessault écrivait avant que n'éclate l'épizootie dite de «la vache folle». De sorte que les vaches de Marchessault étaient peut-être bien folles à leur manière, mais elles ne remettaient certainement pas en question l'existence même de la race humaine. Ce que le professeur dont il est question ici - qui s'identifie lui aussi aux vaches, mais aux vaches folles - a de singulier est précisément de se représenter comme un danger pour l'humanité tout entière. L'étudiant est pour lui un ange exterminateur qui pourrait, en l'éliminant, assurer la survie de la race humaine. Le fait qu'il s'agisse d'un professeur de littérature n'a, bien entendu, rien de rassurant, puisque son discours repose sur un constat d'échec dont il inverse les termes. Plutôt qu'un étudiant qui dit n'importe quoi, nous avons un prof qui dit n'importe quoi : «Je suis ton miroir.» (63)

Bien qu'elle n'y ait jamais consacré qu'une part brève de sa carrière, Marie-Claire Blais est restée fidèle à l'écriture dramatique qu'elle destine généralement à la radio. Noces à midi au-dessus de l'abîme ${ }^{10}$ a toutefois été écrit pour la scène et créé par l'Eskabel en novembre 2005 dans une mise en scène de Jacques Crête. On connaît l'intérêt de Crête et la tradition de l'Eskabel en matière de thêâtre expérimental, et l'on ne s'étonnera guère dès lors du caractère un peu étrange de cette pièce à l'écriture désincarnée (au sens où ne s'y incarnent ni la présence physique des acteurs ni

$$
++
$$

9 Il s'agit de l'incipit de «Les vaches de nuit», paru dans Triptyque lesbien, Montréal, les Éditions de la Pleine Lune, 1980. Voir p. 83. 10 Marie-Claire Blais, Noces à midi au-dessus de l'abîme et autres textes dramatiques, Montréal, Boréal, 2007, 219 p. 
le sentiment d'un espace précisément défini) qui conserve, par là, les attributs essentiels d'un thêâtre écrit pour l'oreille plutôt que pour l'œil. La pièce est accompagnée de deux autres, Désirs et Petites éternités perdues, publiées sans mentions de création.

La pièce éponyme se déroule dans un pays tropical, près de l'océan, les noces de Martin et Éric devraient être un moment de réjouissances, mais plane un danger dont on ignore la source. Autour d'eux, mais pas toujours avec eux, se trouvent plusieurs couples, visiblement en vacances. Aussi la pièce est découpée en scènes brèves qui juxtaposent l'histoire de ces couples. Tous tentent de surmonter une crise et tous sont réunis à leur vie antérieure par un téléphone portable qui fait ainsi intrusion dans l'univers idyllique de cette plage. Le danger approche néanmoins et quand les personnages, vêtus de blanc au début de la pièce, sont devenus des personnages vêtus de noir, l'on comprend qu'il est survenu une catastrophe, mais on ne saura pas au juste quoi. Dans Désirs, un couple dans la soixantaine qui s'est peutêtre installé dans les Keys en Floride pour fuir ce qui fut une vie plus mondaine vit une relation trouble avec un jeune homme. «Le drame est celui de trois êtres que trouble la jalousie ou l'envie, qui sont déchirés l'un par l'autre» (80), annonce la présentation. L'on sent bien, en effet, que le jeune homme et son désir de vivre heurtent de front la vie paisible de Geneviève et Gabriel au seuil de la vieillesse. Car Gabriel voit l'âge comme le moment de céder aux derniers désirs alors que Geneviève au contraire le considère comme le moment de terminer les projets entrepris autrefois. Il y a là, certes, une réflexion sur le sens de la vie, au moment où elle achève. Petites éternités perdues met en scène trois générations de femmes. La plus âgée, Rachel, fut jadis une musicienne de renom, mais la maladie la cloue désormais à l'inactivité. Elle a besoin de soins spécialisés que ne peut plus lui assurer sa fille Suzanne, laquelle refuse cependant de renoncer malgré les exhortations de sa sœur et de sa fille. Dans un dialogue qui reste tout en douceur percent néanmoins les conflits entre ces femmes. Devenue médecin, Élisabeth reproche à sa mère et à sa tante la vie facile dont elles ont pu profiter: «je ne comprends pas les femmes oisives, toi et Andrée, je ne comprends pas.» (177) Pourtant celles-ci se voient d'abord comme des artistes, ayant mis leur art provisoirement de côté au profit de leur vie familiale. Élisabeth n'est pas dupe de cet art prétendu qui n'est qu'une œuvre de dilettantes et que la maladie de Rachel retarde indéfiniment, servant ainsi de prétexte à une oisiveté prolongée.

Dans les trois textes de Marie-Claire Blais, les personnages sont des artistes (acteurs, peintres, musiciens) parvenus à un moment de leur vie où l'art est en suspens, provisoirement ou définitivement. L'action se déroule dans un espace où la nature est généreuse, celui du Sud des vacanciers, que l'auteure définit comme «le lieu qui représente les émotions des personnages, l'atmosphère qu'elles portent en elles-mêmes» (159). Or, dans chaque texte, cette nature apparaît comme un refuge, où sont réunis des personnages qui fuient le monde, leur famille, leurs enfants, leurs désirs, mais ce refuge n'est pas assez puissant pour offrir une protection durable. La haine rattrape les invités aux noces de Martin et Éric; Gabriel se noie; Suzanne cache sa surconsommation de médicaments. La situation dramatique oppose la sérénité que paraissent avoir atteint les personnages à leurs démons intérieurs. La vie factice 
des vacanciers et celle des retraités sont ici confondues voire dénoncées. Car dans les trois textes, c'est la jeunesse et la vie, et donc l'avenir, qui triomphent.

Dans tous les textes dont il a été question dans cette chronique, la question de la transmission fait surface de manière itérative. Toutefois, cette question me semble engager chaque fois une réflexion sur l'identité générique de l'auteur dramatique, qui hésite à se concevoir comme un écrivain ou qui s'affirme comme tel. Ce n'est pas le cas ici. Forte d'une œuvre déjà fermement reconnue, Louise Dupré publie un premier texte dramatique, Tout comme elle, créé à l'Usine C en janvier 2006, dans une mise en scène de Brigitte Haentjens. Comme écrivaine, Dupré n'a plus à affirmer son identité. La filiation se fait d'abord à l'ensemble de l'œuvre de l'auteure qui, depuis plus de vingt ans, explore, dans ses romans, poèmes et essais, les diverses facettes de l'identité féminine, mais elle est également mise en jeu dans les questions que soulève la pièce.

Le texte est désigné comme une pièce en quatre actes, mais il s'agit plutôt de quatre parties composées de douze tableaux chacune, chaque tableau tenant sur une seule page. La notion d'acte suppose en effet le déroulement d'une action dramatique, entre une situation initiale (l'exposition) et une situation finale (le dénouement). Or l'action, en ce qu'elle est transformation, est ici absente, car ce qui intéresse l'auteure est, au contraire, la répétition du même, dans un temps infini. Car la pièce est d'abord et avant tout une longue réflexion sur "la généalogie infinie des filles et des mères» (9), sur ces «filles qui sont des mères et [ces] mères qui sont des filles» (9) et qui, néanmoins, parviennent difficilement à se parler. De sorte que les dialogues sont réduits au strict minimum et la pièce avance plutôt par monologues croisés ou alternés. Le prétexte est une rencontre brève, autour d'une tasse de thé par exemple. L'on entend la fille réfléchir à voix haute: «j'aurais envie de lui dire quelle femme je suis quand je ne suis pas sa fille. Mais elle ne m'entendrait pas.» (36) Puis l'on entend la mère: «Je n'ai jamais su comment lui parler.» (18) La mère et la fille de la première partie sont-elles les mêmes que celles de la dernière ou sontelles des figures différentes, juxtaposées simplement pour désigner l'identité et le même? On se souviendra que la mise en scène de Brigitte Haentjens convoquait cinquante comédiennes, mais on pourrait aussi imaginer la chose autrement. Car ce qui importe dans la réflexion de Dupré est bien de montrer comment cette rupture résulte d'une construction ancestrale et qu'elle doit être envisagée comme une contrainte. Les personnages partagent leur identité de mère et de fille à la fois, mais elles ne peuvent être l'une et l'autre à la fois devant cet autre soi-même qui précède ou qui suit: devant sa mère, une fille reste une fille et n'est pas une autre mère. L'inverse est tout aussi vrai. On le voit, si Tout comme elle se lit comme une prose minimaliste, tant la version donnée à la lecture est dénuée de toute référence à la scénographie (nous sommes ici aux antipodes de ce que propose Robert Lepage) voire à l'espace lui-même, le recours au genre dramatique est néanmoins essentiel, appelé par le propos. En effet, seule la scène permet la représentation simultanée des deux personnages dans leur solitude, ramenant la représentation du temps à celle d'une 
infinie et insupportable répétition. De sorte que la rencontre entre les deux femmes ne pourra advenir que le jour où mères et filles auront trouvé une manière de «briser la chaîne des générations » (65), qui interdit toute rencontre authentique. Ce jour-là, peut-être pourront-elles rompre la douloureuse solitude qui les habite et devenir simplement des femmes qui diront: «j'avance, pas à pas, vivante, vivante de ma seule vie, comme dans un amour désormais libre de toute éternité» (74). 